\title{
A Reformed Resistive Sensor Measuring System Jiandang $\mathrm{GAO}^{\mathrm{a}}$, Yun TENG ${ }^{\mathrm{b}}$ and Yanping CUN* \\ 1Yunnan Open University, Kunming, 650223, P.R.China \\ a jiandang_gao@163.com, b yteng_you@163.com, * cunyanping@163.com
}

Keywords: resistive sensors. measurement system. single-chip

\begin{abstract}
Resistive sensor is widely used in every aspect of production and life, and it has many good qualities, like wide resistance range, nonlinear resistance change and so on. Resistance sensor measurement system is an important step of design and improvement of the property of resistive sensor. A reformed resistive sensor measuring system was discussed in this paper, and 52 series single-chip computer control system was used as a key part. Analysis results shows that it can well realize automatic $\Omega 10-100 \mathrm{~m} \Omega$ resistance measurements.
\end{abstract}

\section{Introduction}

There are many kinds of resistive sensors, and they have wide application [1, 2]. The sensor's resistance changes as the physical quantity [3], such as displacement, deformation, stress, gas, temperature, humidity, etc.. Resistive sensor has the following characteristics: wide resistance range, nonlinear resistance change, large discreteness for the same kind of resistive sensors [4] (voltage dependent resistor, photosensitive resistance, etc).

Resistance sensor measurement system requires measuring resistance has wide tolerance range, range automatic conversion and fast response speed[5]. A reformed resistive sensor measuring system was built in this paper. 52 series single-chip computer control system can realize automatic $\Omega$ 10-100 $\mathrm{m} \Omega$ resistance measurements. Single chip microcomputer as the core controller measurement system, complete the automatic range switching, data measuring, data processing, data output display control function.

\section{Hardware system}

Hardware system was shown in Fig.1. When measurement system starts, will first test the values of the parameters by the ADC transformation into the single chip microcomputer to the system, carries on the range. If the range is too small, then gradually expand it and the range is too large then reduces it. Until to measuring state during the switch to the appropriate range of MCU startup look-up table procedures, to find corresponding display data into measurement data into the LCD display circuit.

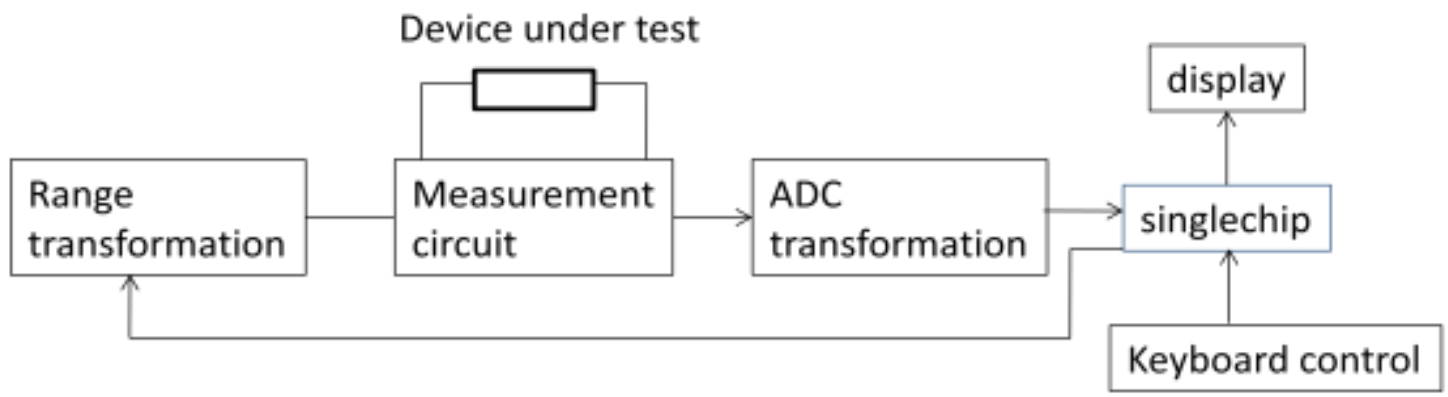

Fig.1 Hardware system 


\section{Measurement circuit}

Measurement circuit was shown in Fig.2. CD4051 is a 8-channels digital control analog electronic switch which choose three binary number input from address $A B C$ as eight channel selection. INH is the enable control. When $\mathrm{INH}=0$, channel selection is valid, and when $\mathrm{INH}=1$, all channels are cutoff. Operational amplifier A is an inverse ratio operation circuit and amplifier B is a voltage follower to improve the load capacity.

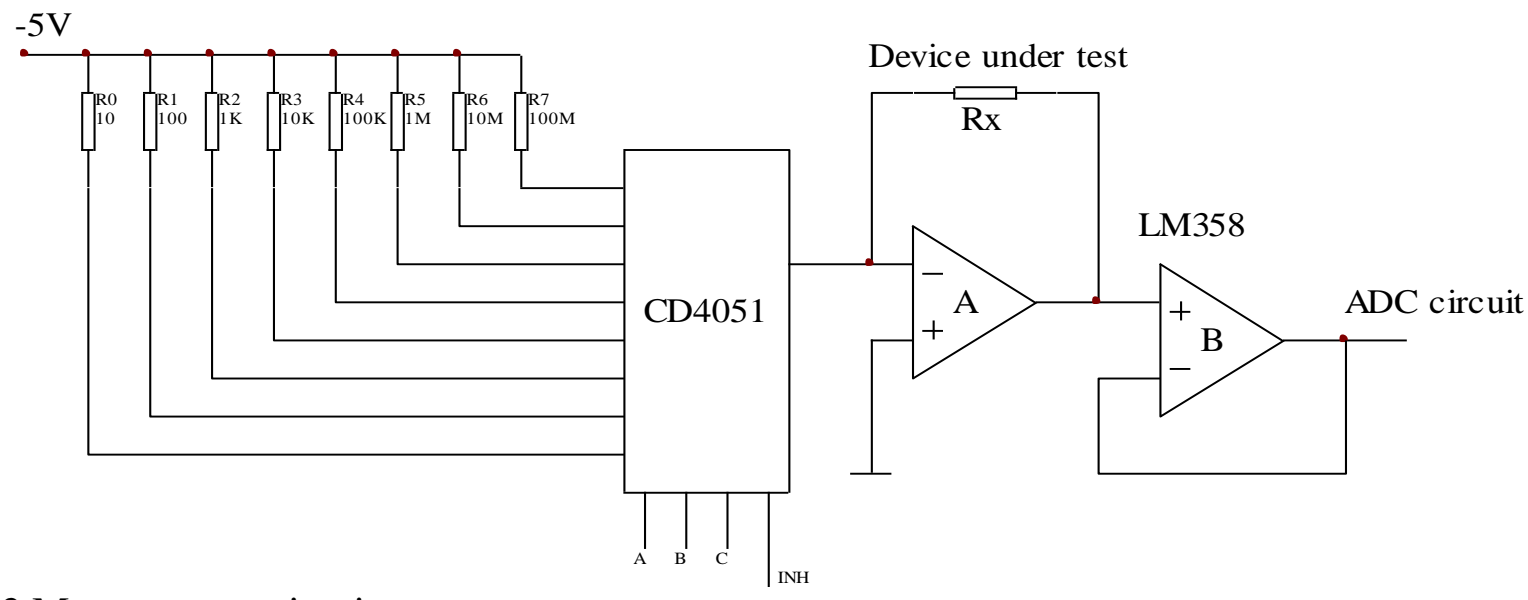

Fig.2 Measurement circuit

\section{Data Collection}

Data collection was done by ADC0809, a 8-bit analog-digital converter. ADC0809 can divide analog voltage output from operational amplifier B into $2^{8}=256$ grades. System would take 250 grades and converts analog to digital quantity. When data collection starts, the single chip firstly read data of ADC0809 to do the judgment. The single chip will control and adjust the range to be appropriate and send data to table look-up program, the send the resistance of the corresponding values to display to realize automatic testing and display.

\section{Single Chip}

AT89C52 was used as a core component of the control system, and single chip system is mainly to complete the range switch, conversion (look up table), output display control function. AT89C52 was made by ATMEL at 2003, and it Is compatible with 8051, ISP programming and watchdog functions, single-chip switching speed, storage capacity meet the system rEquirements.

\section{Displayer}

Liquid crystal display shows strong function, low voltage, low power consumption and low cost. $12864 \mathrm{C}-1$ has a variety of interface mode, such as 4/8 bit parallel, $2 / 3$ serial interface lines, and its internal part contains national standard first class and second class of the simplified Chinese character bitmap graphics LCD module. Besides, its display resolution is $128 \times 64$, and it is built in with 8192 $12816 * 16 * 16$ point of Chinese characters and eight ASCII character set. $12864 \mathrm{c}-1$ module was used, since its simple Interface and convenient operating instructions. Additionally, it can display $8 *$ 4 line $16 * 16$ dot matrix Chinese characters and constitute the human-computer interaction in Chinese and graphical display graphics.

\section{Work flow of measurement system}


Work flow of measurement system was shown in Fig.3. Operational amplifier output voltage in the measurement circuit must be between $0.5 \mathrm{~V}$ to $5 \mathrm{~V}$. The output voltage is less than $0.5 \mathrm{~V}$ range is too large range shall be reduced, the output voltage is greater than $5 \mathrm{~V}$ range too small should be increased. For example, the measured resistance $\mathrm{Rx}=580 \Omega$, start the measurement circuit, and single chip send data to $\mathrm{CD} 4051$ address. $\mathrm{ABC}=000$, then channel 0 was chosen, the computation of proportion coefficient is

$$
A_{\mathrm{u}}=-\frac{\mathrm{u}_{\mathrm{A}}}{-5}=\frac{R_{x}}{R_{0}}=\frac{580}{10}=58 \quad u_{A}=-58 \times(-5)>5 V
$$

Operational amplifier A was into nonlinear area, and the output voltage is the maximum value. When $u_{A}>5 \mathrm{~V}$, it means the range small, and single chip will plus 1 to address code to increase the range.

$\mathrm{ABC}=001$,channel 1 was chosen and computation of proportion coefficient is $580 / 100=5.8$, $u_{A}=-5.8 \times(-5)>5 \mathrm{~V}$, range was still too small, and single chip will plus 1 to address code to increase the range again.

$\mathrm{ABC}=010$, channel 2 was chosen and computation of proportion coefficient is $580 / 1000=0.58$, $u_{A}=-0.58 \times(-5)=2.9<5 \mathrm{~V}$, range was right, and single chip would operate the table lookup program, then find the send the resistance of the corresponding values to display. It would keep a reading time and do the data collection again.

Sensor's resistance would change as the change of physical quantity, If resistance decreases, less than $0.5 \mathrm{~V}$, it means the range is too large, and single chip will minus 1 to address code to reduce the range.

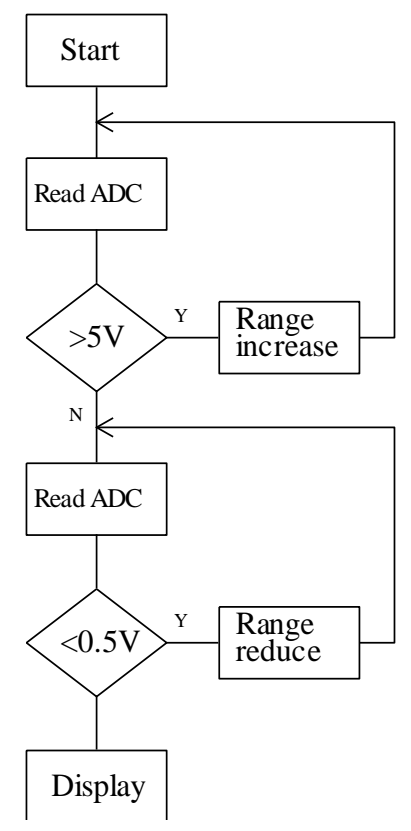

Fig. 3 Work flow

\section{Conversion Relation}


Delta of the system is $0.02 \mathrm{mV}$, and there is a datum form with 250 lines for $0-5 \mathrm{~V}$ range. Output voltage of operational amplifier, $u_{A}$, and measured resistance $R_{x}$ have a conversion relation:

$$
u_{A}=-\frac{R_{x}}{R_{i}}(-5)=5 \frac{R_{x}}{R_{i}} \quad R_{x}=\frac{1}{5} R_{i} u_{A} \quad i=0,1,2, \ldots 7
$$

When $u_{A}=2.9 \mathrm{~V}$, the corresponding range is $\mathrm{ABC}=010, R_{i}=1000 \Omega$.According to Eq. 2 ,

$$
R_{x}=\frac{1}{5} R_{i} u_{A}=\frac{1}{5} \times 1000 \times 2.9=580 \Omega \text {. }
$$

Microcontroller programming can maneuver algorithm also can use the table lookup method. Although table lookup method will take a large memory, it has a rapid response speed. Thus, table lookup method was used in this system. Moreover, factors like the discrete circuit component and circuit layout could cause nonlinear system error, and this system is able to test 250 points and the error of measurement can be eliminated by using the method of add and subtract revised method.

\section{Experimental measurement results}

The measurement results were listed in table 1. It can be found that the resistance value varied as input analog quantity, and the measurement resistance was listed in table 2.

Tabel 1 Measurement results

\begin{tabular}{cccc}
\hline & $\begin{array}{l}\text { ADC 0809analog quantity } \\
\text { imput }(\mathrm{mV})\end{array}$ & $\begin{array}{l}\text { ADC } \\
\text { 0809digital quantity output }\end{array}$ & resistance display $(\Omega)$ \\
\hline 0 & 0 & 00000000 & 0 \\
1 & 20 & 00000001 & 4 \\
2 & 40 & 00000010 & 8 \\
3 & 60 & 00000011 & 12 \\
4 & 80 & 00000100 & 16 \\
5 & 100 & 00000101 & 20 \\
6 & 120 & 00000110 & 24 \\
7 & 140 & 00000111 & 28 \\
$\ldots$ & & & 1000 \\
\hline
\end{tabular}

Table 2 Tested resistance $(100 \Omega)$

\begin{tabular}{cl}
\hline Tested resistance $(\Omega)$ & Practical measured values $(\Omega)$ \\
\hline 10 & 9.60 \\
30 & 28.00 \\
50 & 49.00 \\
70 & 68.70 \\
90 & 88.70 \\
\hline
\end{tabular}

Based on a large amount of measurement data, it can be found that measurement error is opposite bigger when the resistance is small. As for system error, adding modification value in program can minimize measurement errors. 


\section{References}

[1] Y. W. Yu. Sensor principle and engineering application. Xi'an, Xian university of electronic science and technology press (2007).

[2] R. Pallas-Areny, J.G. Webster. Sensors and signal conditioning, New York, NY : Wiley (2001).

[3] T. Takeuch. Oxygen sensors. Sensors and Actuators, 14 (1988), p.109.

[4] S.Y. Yurish. Sensors and Transducers: FrEquency Output Versus Voltage Output. Sensors \& Transducers Magazine (S\&T e-Digest) 49(2004), p. 302-305

[5] G. D. Luan. Sensor and its application. Xi'an, Xian university of electronic science and technology press (2002). 